\title{
Numerical modelling of thermal decomposition processes and associated damage in carbon fibre composites
}

\author{
R D Chippendale, I O Golosnoy and P L Lewin \\ University of Southampton, Tony Davies High Voltage Laboratory, Highfield, Southampton, \\ SO17 1BJ, UK \\ rd.chippendale@soton.ac.uk
}

\begin{abstract}
.
Thermo-chemical degradation of Carbon Fibre Composite materials (CFCs) under intensive heat fluxes has been modelled. The model couples together heat diffusion, polymer pyrolysis with associated gas production and convection through partially decomposed CFCs, and changes in transport properties of the material due to the damage. The model has been verified by laser ablation experiments with controlled heat input. The numerical predictions indicate that the thermal gas transport has a minimal affect on the decomposition extent. On the other hand, the model shows that the internal gas pressure is large enough to cause fracture and delamination, and the damage extent may go far beyond the decomposition region as witnessed from experimental verification of the model.
\end{abstract}

Keywords:

Carbon fibre composites, pyrolysis, modelling, fractional steps method

\section{Introduction}

Carbon Fibre Composites (CFCs) are currently finding more and more applications as a structural material, especially within the aerospace industry. It is not surprising since CFCs are comparatively equal in strength to the more traditionally used materials like aluminium alloys, whilst being considerably lighter. However, CFCs are comparatively poor electrical and thermal conductors. They cannot dissipate an excessive heat quick enough and they also undergo through very different thermo-physical degradation. These differences become important when considering the interaction of a lightning strike with an aircraft made from CFCs. The typical damage reported for unprotected CFCs panels due to a lightning strike are polymer degradation, ply de-laminations and splintering of carbon fibres [1]. This is quite similar to the damage reported after the fire exposure [2] or laser drilling / cutting of CFCs using $\mathrm{CO}_{2}$ and $\mathrm{Nd}$ :YAG lasers [3], which is not very surprising since the damage in all these cases has a thermal 
nature. Nevertheless, there are noticeable differences in the damage for these cases. The surface fire damage mainly removes the polymer leaving fibre skeleton exposed [2]. The drilling process injects so much energy into the material that both fibres and polymer are taken away without significant fracture or ply delamination [4][5]. This indicates that the lightning strike damage is somewhere between these two cases, which can be successfully modelled without considering details of the gas filtration through the partially decomposed CFCs [2]. Although previous studies [6][7] have investigated the damage due to lightning strikes, minimal attention has been given to the filtration of gas production. The latter is primarily important for predictions of the reported cracking and de-lamination, plus it is unclear how much thermal energy is actually transported by the gas and what effects it may have on the extent of damage region.

This study develops a comprehensive numerical model to predict the thermal chemical degradation processes through which CFCs degrade and the model fills the gap between previous models [6][7]. It is worth noting that a lightning strike generates two thermal inputs, the surface heat flux from the plasma arc and the volumetric joule heat from the injected current. It is also a complex coupled thermalelectric phenomenon and for the model verification simpler situations need to be considered. A slightly defocused laser beam can deliver a power density similar to a lightning strike in a controlled way with only surface heat flux. Similar to lightning, this heat source generates a thermal profile within the CFC which drives decomposition. Generally, two main thermal degradation mechanisms have been reported for CFCs, polymer pyrolysis and the carbon fibre sublimation [8][2]. The pyrolysis reduces the polymer to carbon rich chars and gases. The produced gases then filtrate through the formed gaps between the carbon fibres. It is the production of these gases and their filtration through the decomposing CFCs, which controls the pressure built-up and causes de-lamination and fracture. The aims of this study are to investigate the kinetics of gas production and the effects of gas transport on damage.

Powerful lasers have been previously used to cut or drill holes in CFC samples [3][5][8][9][10][11][12]. The damage mechanism outlined in these studies appear analogous to observed lightning strike damage, i.e. polymer pyrolysis and cracking within CFC, which confirms the thermal mechanism of decomposition for all these processes. Laser ablation is a preferable choice of verification experiment, because the power input is well characterised and it removes most uncertainties related to boundary conditions. It was used to verify the model described in sections 2 and 3 . Details of the verification experiment are given in section 4 .

\section{Materials Model Framework}

Before a numerical model can be developed, a numerical framework to represent CFC needs to be carefully considered. The choice of framework is dependent on the physical processes under investigation 
and can have a significant impact on the simulation efficiency. Once the modelling framework has been outlined the method by which material properties are described within this framework are detailed.

\subsection{Homogenization}

Detailed analysis of thermo-chemical degradation would require a separate discretization of the matrix (polymer) and fibres. However it's not feasible to directly represent each $6 \mu \mathrm{m}$ diameter fibre within a typical panel size of $1 \mathrm{~m}^{2}$ due to the large number of mesh elements which would be required.

Nevertheless, the most important features of the decomposition process may be predicted by considering CFCs as a homogeneous anisotropic material [13]. The fundamental simplification of the homogenisation approach is that the complex internal structure of a piece of CFC can be numerically represented by a simple block, which has the same material properties as the bulk CFC panel and it can be applied when the size of the modelling elements significantly larger than the fibre diameter. The main issue is the definition of an appropriate discretization size.

Carbon Fibre Composite materials are formed from plies, consisting of long strands of conductive carbon fibres encapsulated in a poorly conductive polymer (epoxy) matrix. This internal ply structure creates a highly anisotropic ply. Whilst laying up plies, the alignment of fibres between the plies can be varied to obtain the required mechanical properties. This further complicates the bulk material properties. Throughout this study a local axis is defined for each ply, where the direction parallel to the fibre is designated 1, and direction 2 is perpendicular to the fibre in the plane of the ply. Direction 3 is taken as the through thickness direction of the CFC sample, and is therefore the rotational axis over which the other two directions rotate when switching from one ply to another. The rotation of the local axis for each ply, is given with reference to a global axis. In terms of discritization, each ply has to be modelled as anisotropic layer, but generally only a dozen elements are required to represent the through-thickness of each ply, to be sufficient to generate realistic predictions although it contains hundreds of fibre layers.

This study uses the finite volume approach to discreitize the geometry by dividing it up into small mesh elements, which each element representing a volume, $\mathrm{V}_{\text {cell. }}$. When the decomposition front propagates through the CFC plaque, individual model cells may contain a sharp decomposition front and consist of quite different structures before and after the front. It does not strongly affect the validity of the homogenization approach since heat input into the cells mainly goes to the solid part with a high specific heat. In this sense, decomposition mainly affects heat transport via changes in transport properties. When using the homogenisation approach, it is important to be able to accurately predict the bulk CFC properties as a function of the polymer degradation. 


\subsection{Effective Thermal Properties}

There are many published methods on determining the bulk material properties of composites [14][15][16]. All of them require knowledge of the volume fraction of the each species (polymer, carbon fibre, char and gas) present within the bulk material. The volume fraction $\left(\Phi_{i}\right)$ of each species $(i)$, within each mesh element is defined as

$$
\Phi_{i}=\frac{V_{i}}{V_{\text {cell }}}
$$

Equation 1

where the volume each species occupies $\left(V_{i}\right)$ is given with reference to the mass of each species $\left(m_{i}\right)$ and the constant density of each species $\left(\rho_{i}\right)$ by considering

$$
V_{i}=\frac{m_{i}}{\rho_{i}}
$$

Equation 2

This method is only applicable for solid phases which are considered to be incompressible. The gas fraction the volume is calculated by determining the remaining volume within each cell which is not occupied by a solid phase

$$
V_{g}=V_{c e l l}-\left(V_{c h}+V_{p}+V_{f}\right)
$$

Equation 3

Where the subscripts "ch", "f", " $p$ " and " $g$ " refer to char, fibre, polymer and gas respectively. Having calculated the volume occupied by the gas, the gas volume fraction can then be calculated by Equation 1. Using the volume fraction of each species, the bulk heat capacity of the element at constant volume $\left(\rho C_{V}\right)$ is calculated by using the series rule of mixtures,

$$
\rho C_{V}=\sum_{i=f, p, c h, g} \Phi_{i} \rho_{i} C_{V i} \quad \text { Equation } 4
$$

The bulk thermal conductivity tensor in principal axes $(1,2,3)$ is given by

$$
\kappa=\left(\begin{array}{ccc}
\kappa_{1} & 0 & 0 \\
0 & \kappa_{2} & 0 \\
0 & 0 & \kappa_{3}
\end{array}\right) \quad \text { Equation 5 }
$$

The conductivity tensor in global $(\mathrm{x}, \mathrm{y}, \mathrm{z})$ coordinates can be recalculated using a standard transformations. The bulk conductivity along direction $1\left(\kappa_{1}\right)$ is given by the Series Rule of Mixtures [15]

$$
\kappa_{1}=\Sigma_{i=f, p, c h, g} \Phi_{i} \kappa_{i} \quad \text { Equation } 6
$$

As the decomposition progresses, the voids start to form in the polymer matrix and Equation 6 is not strictly valid. But the main contribution to the conductivity comes from the fibres (since the fibre 
conductivity is 2 orders of magnitude greater than that of epoxy) which stay undamaged and Equation 6 provides a reasonable approximation to the real case. This phenomenon has been observed during laser processing of CFCs $[8,10]$.

The polymer degradation more greatly affects the conductivity normal to the fibres. Although reasonable and accurate models have been suggested for two-component systems with uniformly distributed fibres [16], the damaged multi-component system (fibres, matrix, char, gas) cannot be modelled precisely. The thermal conductivity in the remaining two directions (direction 2 and 3) are assumed to be isotropic and can be approximated by the Parallel Rule of Mixtures,

$$
\kappa_{3}=\kappa_{2}=\left(\frac{\Phi_{f}}{\kappa_{f}}+\frac{\Phi_{p}}{\kappa_{p}}+\frac{\Phi_{g}}{\kappa_{g}}+\frac{\Phi_{c h}}{\kappa_{c h}}\right)^{-1}
$$

Equation 7 provides a reasonable approximation even for the model cells covering the decomposition front.

\subsection{Properties of Constituents}

An experimental study has been conducted to determine the reaction kinetics required to represent the polymer degradation [17] assuming the temperature dependent reaction

$$
\begin{array}{ll}
\frac{d m_{p}}{d t}=-R \quad \text { Equation } 8
\end{array}
$$

where $\mathrm{R}$ is the reaction rate given by the Arrhenius equation

$$
R=A m_{p}\left(\frac{m_{p}(t)-m_{p}\left(t_{\infty}\right)}{m_{p}\left(t_{0}\right)}\right)^{n} \exp \left(-\frac{E_{a}}{\mathfrak{R} T}\right) \quad \text { Equation } 9
$$

Where $n, A, E_{a}$ and $\mathfrak{R}$ are the reaction order, the pre-exponential factor, the activation energy and the molar gas constant, respectively. The initial and final mass of the polymer are given by $m_{p}\left(t_{\infty}\right)$ and $m_{p}\left(t_{0}\right)$. The following reaction kinetics have been obtained for M21/T700 composite used in this study: $\mathrm{E}_{\mathrm{a}}$ is $181.73 \times 10^{3} \mathrm{Jmol}^{-1}$, $\mathrm{A}$ is $3.15 \times 10^{11} \mathrm{~s}^{-1}$ and $\mathrm{n}$ is 1.344 . The change in enthalpy caused by the pyrolysis has been experimentally measured to be $\left(Q_{d}\right) 0.9 \times 10^{6} \mathrm{Jkg}^{-1}[17]$. As well as kinetic decomposition (pyrolysis), the epoxy (polymer) and carbon fibre within the CFC could undergo phase changes. Measurements indicate the glass transition temperature $\left(\mathrm{T}_{\mathrm{g}}\right)$ for typical epoxies are between 300 and $400 \mathrm{~K}$, although these values depend on pressure and heating rates [18] (manufacture quote $\mathrm{T}_{\mathrm{g}}$ for M21 to be $383 \mathrm{~K}$ ). This study neglects the dependence of thermal and mechanical properties due to glass transition and pressure, keeping in mind a possible $100 \%$ increase in thermal conductivity of epoxy [18]. 
The specific heat capacity of epoxy is less affected by $\mathrm{T}_{\mathrm{g}}$ [18]. Carbon fibres sublimate at around $3500 \mathrm{~K}$ [8] and such temperatures rarely are achieved and so are not considered further.

The approximate thermal properties have been obtained from literature [19][20], are defined in Table 1

Table 1 - Material properties used in model

\begin{tabular}{|c|c|c|c|}
\hline Parameter & Symbol & Value & Units \\
\hline Thermal conductivity of polymer & $\kappa_{\mathrm{p}}$ & 0.1 & $\mathrm{Wm}^{-1} \mathrm{~K}^{-1}$ \\
\hline Density of polymer & $\rho_{\mathrm{p}}$ & 900 & $\mathrm{~kg} \mathrm{~m}^{-3}$ \\
\hline Heat capacity of polymer & $\mathrm{C}_{\mathrm{vp}}$ & 2500 & $\mathrm{Jkg}^{-1} \mathrm{~K}^{-1}$ \\
\hline Thermal conductivity of gas $\left(\mathrm{CO}_{2}\right)$ & $\kappa_{\mathrm{g}}$ & 0.025 & $\mathrm{Wm}^{-1} \mathrm{~K}^{-1}$ \\
\hline Density of gas $\left(\mathrm{CO}_{2}\right)$ & $\rho_{\mathrm{g}}$ & 1.997 & $\mathrm{kgm}^{-3}$ \\
\hline Heat capacity at constant pressure for gas $\left(\mathrm{CO}_{2}\right)$ & $\mathrm{C}_{\mathrm{vg}}$ & 720 & $\mathrm{Jkg}^{-1} \mathrm{~K}^{-1}$ \\
\hline Thermal conductivity of fibre & $\kappa_{\mathrm{f}}$ & 9.37 & $\mathrm{Wm}^{-1} \mathrm{~K}^{-1}$ \\
\hline Heat capacity of fibre & $\mathrm{C}_{\mathrm{vf}}$ & 794 & $\mathrm{kgm}^{-3}$ \\
\hline Density of fibre & $\rho_{\mathrm{f}}$ & 1760 & $\mathrm{kgm}^{3}$ \\
\hline Molar gas constant & $\mathrm{M}$ & 0.044 & $\mathrm{kgmol}^{-1}$ \\
\hline Thermal conductivity of char & $\kappa_{\mathrm{ch}}$ & 0.2 & $\mathrm{Wm}^{-1} \mathrm{~K}^{-1}$ \\
\hline Density of char & $\rho_{\mathrm{ch}}$ & 1300 & $\mathrm{kgm}^{-3}$ \\
\hline Heat capacity of char & $\mathrm{C}_{\mathrm{vch}}$ & 1589 & $\mathrm{Jkg}^{-1} \mathrm{~K}^{-1}$ \\
\hline
\end{tabular}

The model assumes that all material properties are constant with temperature. The initial volume fractions of polymer and fibres have been defined as follows: $\phi_{\mathrm{p}}\left(\mathrm{t}_{0}\right)$ is equal to 0.4 and $\phi_{\mathrm{f}}\left(\mathrm{t}_{0}\right)$ is equal to 0.6 according to M21/T700 composite specification. These initial volume fractions assume there are no void present within a virgin panel of CFC. Finally, the produced gas is assumed to be an ideal, the gas pressure can be calculated by

$$
P=\frac{m_{g} \mathfrak{R} T}{M V_{g}}
$$

Equation 10

where $M$ is the molar gas constant for the specified gas, $V_{g}$ is the volume occupied by the gas within each mesh element, the molar gas constant is $\mathfrak{R}$ is $8.31 \mathrm{JK}^{-1} \mathrm{~mol}^{-1}$.

\section{Numerical Model Formulation}

Using the above framework to describe the CFCs properties, the governing equations for a homogeneous material to represent the physical processes of the decomposition can be outlined. 


\subsection{Thermal Transport}

There are several equivalent formulations for diffusion-convection heat transport [21]. The proposed model deals with constant volume elements within a variable pressure environment. The most convenient way to model the temperature field in the CFC is by considering the conservation of internal energy. The heat transport through a material as a function of time $(t)$ is given by

$$
\begin{array}{ll}
\frac{d\left(\rho C_{v} T\right)}{d t}=\nabla(\kappa \nabla \mathrm{T})+\frac{Q_{d}}{V} \frac{d m_{p}}{d t}-\nabla\left(v_{g}\left[\rho_{g} C_{v} T+P\right]\right) & \text { Equation } 11
\end{array}
$$

where $C_{V}, \rho$ and $\kappa$ are the effective specific heat capacity of the cell at constant volume, density $\rho$ and thermal conductivity $\kappa$. The temperature $(T)$, is given for the entire contents of a mesh element. Inherent within this expression is the assumption that all species are in thermal equilibrium within each element volume. The filtration velocity, $v_{g}$, is further defined in Equation 14.

The left-hand side (LHS) term in Equation 11 considers the change in internal energy as a function of time. The first term on the right-hand side (RHS) is a contribution of thermal conduction. The second term on the RHS side is responsible for the energy consumption $\left(Q_{d}\right)$ due to the polymer pyrolysis. The final term on the RHS is a contribution of the gas convection which is defined in terms of the filtration velocity, $v$, and the work done in the variable internal gas pressure field $(P)$.

\subsection{Chemical degradation}

Unlike the thermal transport problem, each constituent chemical species requires its own governing equation. There is no need to consider an equation for the carbon fibre, as it assumed that carbon fibres do not undergo a chemical reaction and that they are solid and immobile. The possible oxidation of carbon fibres are not considered here, since the pyrolysis products escaping from the damaged area block any oxygen supply and the decomposition in air is close to that under an inert atmosphere [4].

The chemical degradation of the polymer due to pyrolysis is given by Equation 8 and 9. Due to the conservation of mass the fractions of gas and char which are produced must equal unity, $d\left(m_{p}+m_{g}+m_{c h}\right)=0$. By introducing the mass fraction of the decomposed polymer, which is now gas or char, $\xi_{\mathrm{g}}$ or $\xi_{\mathrm{ch}}$, respectively, the governing equation for the gas mass in the cell can be written as

$$
\frac{d m_{g}}{d t}=\zeta_{g} R+\nabla\left(v_{g} m_{g}\right)
$$

Equation 12

The first term on the RHS gives the rate at which the mass of gas is produced at particular point in the material. The second term on the RHS represents the mass convection through the decomposing material. The equation for the char is similar to (12), and is expressed as 


$$
\frac{d m_{c h}}{d t}=\zeta_{c h} R
$$

Equation 13

with no convective transport term included in Equation 13, as the char is assumed to be an immobile solid which sticks to the surface of the fibres.

The gas filtration velocity in Equation 12 is taken from the Darcy's filtration law

$$
v_{g}=-\frac{\gamma \nabla P}{\mu \Phi_{g}}
$$

Equation 14

where $\gamma$ and $\mu$ are the permeability and the gas kinematic viscosity respectively. The second term in the denominator represents the porosity (voids). As it is assumed that there are no voids within initial CFCs, the porosity can be equated to the volume fraction of gas. The permeability depends mainly on specific surface area of the decomposing material and can be approximately expressed by the Kozeny Blake equation [22][23][24],

$$
\gamma=\frac{\left(2 r_{f}\right)^{2} \Phi_{g}^{3}}{180\left(1-\Phi_{g}\right)^{2}}
$$

where $r_{f}$ is the radius of a single carbon fibre.

Initial voids generation with $\varphi_{\mathrm{g}}$ tends to 0 and low permeability results in very small filtration velocity $\mathrm{v}_{\mathrm{g}}$ tends to 0 . Due to the large difference in specific volume of solid and gas the model predicts very high pressures. This unphysical behaviour is due to the omission of mechanical effects, such as compression of polymer matrix and its cracking and de-laminations along the fibres to generate large pores and easier gas escape routes, which would reduce the pressure. It is impossible to accurately model such effects using a homogenisation approach, but to avoid generating an over pressure, the permeability given by Equation 15 is modified to include gas pressure effects, i.e.

$$
\gamma=\left\{\begin{array}{c}
\frac{\left(2 r_{f}\right)^{2} \Phi_{g}^{3}}{180\left(1-\Phi_{g}\right)^{2}}, \text { if } P<\sigma_{\max } \\
\gamma_{\min }, \text { if } P \geq \sigma_{\max }
\end{array}\right.
$$

Equation 16

The choice of $\sigma_{\max }$ depends on the failure mechanism. Observations in section 4 suggest that the volume fraction of polymer at which fractures are observed are very small. The gas bubble starts to form in the polymer and the associated stress decays inverse proportional to the distance from the bubble. It indicates that the stresses are high in the polymer but they don't extend to the fibres. Based on this observation, $\sigma_{\max }$ is defined by the tensile strength of polymer. If the gas pressure exceeds the tensile strength of polymer within the CFC $\left(\sigma_{\max }\right)$ then the permeability $(\gamma)$ is held at a constant permeability, $\gamma_{\min }$. The 
minimum permeability $\left(\gamma_{\min }\right)$ can be found from the numerical simulation for every CFC material by determining a self-consistent combination of $\left(\gamma_{\min }, \sigma_{\max }\right)$. In the present study the constant permeability $\left(\gamma_{\min }\right)$ of $6 \times 10^{-22} \mathrm{~m}^{2}$ occurs when the gas pressure exceeds $\sigma_{\max }$ is equivalent to $6 \times 10^{7} \mathrm{~Pa}$.

\subsection{Boundary and Initial Conditions}

For the heat transport both boundary conditions and initial values need to be specified. The initial temperature, $T\left(t_{0}\right)$, is assumed to be uniform and equal to $300 \mathrm{~K}$. The heat flux from the laser beam is modelled by a circular 2D Gaussian function, with the function being defined over the $\mathrm{x}-\mathrm{z}$ plane:

$$
Q_{f l u x}(x, z)=\frac{2 P_{L}}{\pi \Lambda^{2}} \exp \left[-2\left(\frac{\left(x-x_{0}\right)^{2}}{\Lambda^{2}}+\frac{\left(z-z_{0}\right)^{2}}{\Lambda^{2}}\right)\right]
$$

Equation 17

The beam radius is given as $\Lambda$ with the beam centred at $\left(x_{0}, z_{0}\right)$. The peak heat flux from the laser beam is defined by the power output from the laser $\left(P_{L}\right)$, divided by the area under the Gaussian curve. Additionally, all boundaries are subject to black body thermal radiation, with the emissivity of the CFC are taken as $\varepsilon_{\mathrm{cfc}}$ is 1 . A constant ambient temperature $T_{a m b}$ is assumed to be $300 \mathrm{~K}$. The convective cooling has been ignored in the model since its contribution is only a small fraction of radiative losses as been confirmed by several trial runs.

An edge boundary condition is required for Darcy's law (Equation 14) and this is that the pressure at all boundaries is equal to 1 Atm. The initial mass of polymer $\left(m_{p}\left(t_{0}\right)\right)$ and carbon fibre $\left(m_{f}\left(t_{0}\right)\right)$ within a cell is given by the initial fibre volume fraction $\phi_{f}\left(t_{0}\right)$ and the density of each species. They are defined as

$$
m_{f}\left(t_{0}\right)=\phi_{f}\left(t_{0}\right) \rho_{f} V_{\text {cell }}, m_{p}\left(t_{0}\right)=\left\lfloor 1-\phi_{f}\left(t_{0}\right)\right\rfloor \rho_{p} V_{\text {cell }} \quad \text { Equation } 18
$$

It is assumed, that initially there is no char, gas or voids within the CFCs.

\subsection{Numerical Algorithm}

Simultaneous solution of the non-linear Equations 11 to 14 is unpractical, due to their coupled nature. This complication is overcome by applying the fractional step method, which replaces a fully coupled model by a linked chain of equations [25]. The numerical algorithm has been implemented using a segregated solver with the following split: transient heat transfer (Equation 11, diffusion only) chemical reaction (Equation 8, 9, 12 and 13, new estimates for pyrolysis products) - temperature changes 
due to pyrolysis (Equation 11), heat source only and gas pressure estimates) - gas transport and thermal convection (Equation 12), with only convection term in Equation 11). The heat diffusion is solved using fully implicit algorithm. The chemical reaction Equation 8 and 9 are solved analytically. With the new temperature and mass profiles, the pressure estimates are obtained which leads to the filtration velocity. The gas convection sub-model is then solved using an implicit backward Euler scheme. The effective material properties are updated after each sub-step. This sequence is repeated on each time step until the total simulation time is reached. The detail of the implementation of this numerical algorithm is given in [17].

\section{Laser Ablation - Verification Experiments}

This study used a $6 \mathrm{~W}$ pumped diode laser from Coherent [26], which had a Gaussian beam radius $(\Lambda)$ of $1.125 \mathrm{~mm}$. Strips of CFC with dimensions, 50mm x 7mm x 5mm (length, width, height) were cut from a larger panel of M21/T700 composites using a diamond edge tile cutter. The composite panel had 22 plies with fibre direction (local axis 1) in each ply was rotated by 45, 135, 0, 0, 90, 0, 135, 0, $45,45,0,0,45,45,0,135,0,90,0,0,135,45$, degrees with respect to the global $\mathrm{x}$ axis along the length of the samples. There are thin layers of polymer between each ply. The samples were ablated for 10, 30, 60, 90, 120, 150, 180 seconds. Each CFC strip used for three ablation experiments, with beam centres

equally spaced along the length of the samples. It insures that the interaction with neighbouring previously ablated regions were negligible.

\subsection{Analysis of Damage}

The ablated samples were then scanned using X-ray tomography with local resolution of 17.7 microns (details of the $\mathrm{x}$-ray scans can be found in [27]) to investigate the spatial dimensions of the damage. An example of a reconstructed X-ray tomography image, for the 180 seconds ablated CFC sample is shown by a top down view in Figure 1 and a cross section through the middle of the damage region (along fibres) in Figure 2. The X-ray tomography shows three distinct regions. The solid grey regions represents the undamaged material, the grey streaky region depicts where the polymer has been removed, but the fibres still remain, and the black region indicates where there is no material present. 


\section{Top down view}

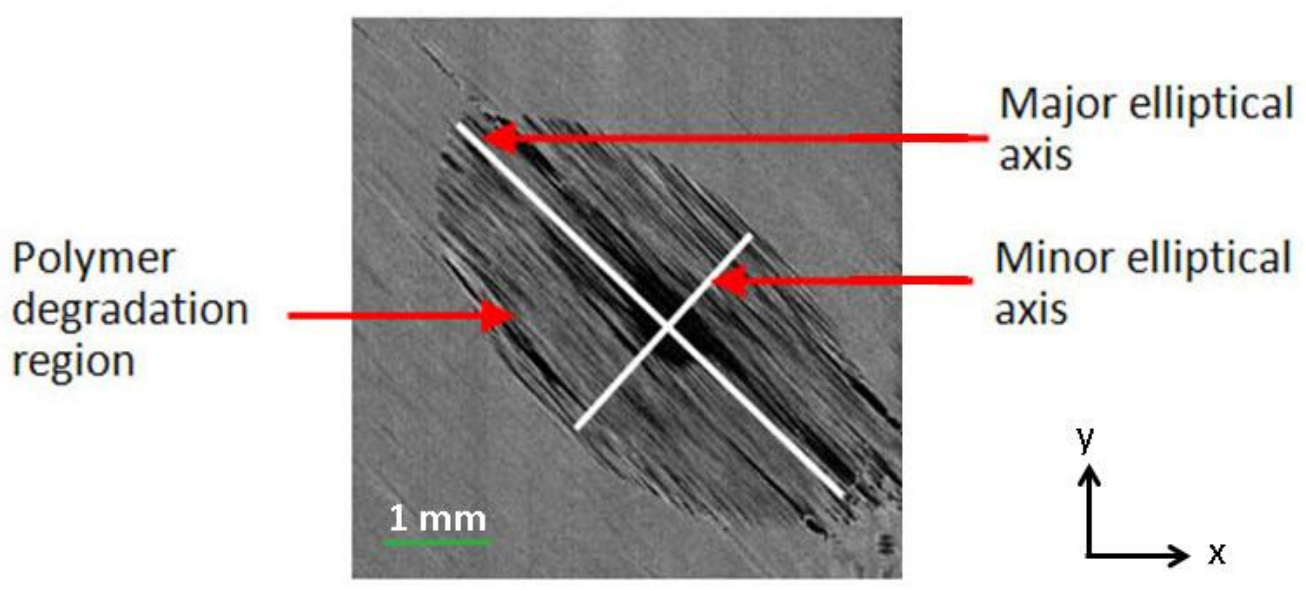

Figure 1: X-ray tomography image of a laser ablated sample with the damaged section orientated in according to the fibre orientation. The damage shown is for an ablation time of 180 seconds.

From Figures 1 and 2 the polymer damaged region resembles a half of ellipsoid, with the major elliptical axis being at 45 degrees to the $\mathrm{x}$ axis. This corresponds to the fibre direction in the top ply. The small dark region in the centre indicates that some fibre removal has also occurred. The cross section slice shown in Figure 2 is taken at the section with maximum damage depth. The observed topology does not show any "undercutting" damage in plies below the top ply, something clearly observed in laser drilling experiments [3]. It is because the heat injection in the present study takes place at the top ply, whereas for drilling experiments [3] the laser directly interact with under surface plies.

\section{Cross section}

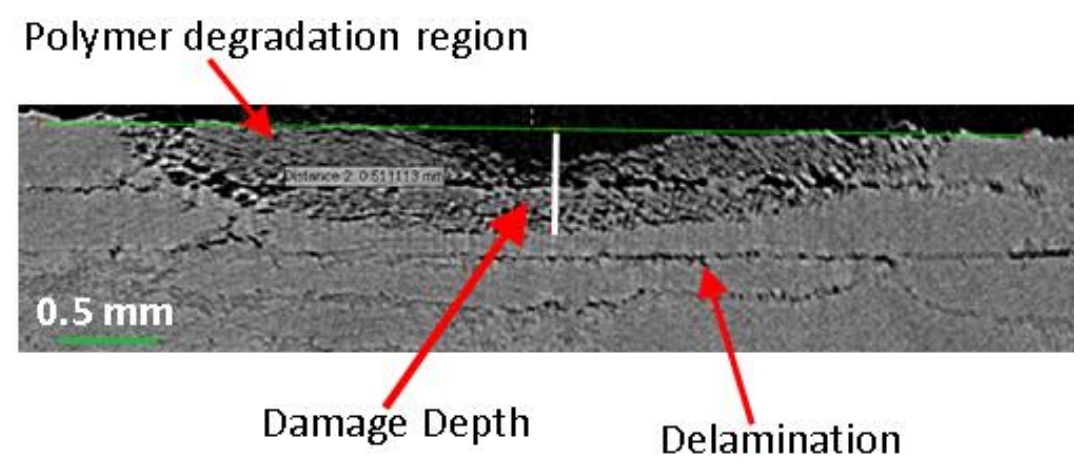

Figure 2: Vertical section of X-ray tomography image for laser ablated sample. The damage shown is for an ablation time of 180 seconds 
In Figure 2, the polymer degradation and the fibre removal region extend to the second ply below the surface. The shape of the decomposed region is elongated in the direction along the fibres since they provide the easier conductive paths. There is also an evidence of cracking and/or de-lamination occurring far below the polymer degradation region. These are believed to be caused by a large internal gas pressure, which occurs due to the gasses produced by the polymer pyrolysis.

The experiments have helped define basic requirements for the model, namely;

1) To determine the typical maximum heat flux when the pressure effects can be neglected and the damage is solely due to chemical reaction.

2) To establish whether the fracture / delamination region be easily linked with the calculated pressure profile or whether an additional mechanical model is needed.

3) To investigate if gas production and transport affect only the pressure profile or if they also contribute to the temperature profile via convection.

4) To establish the importance of the anisotropy on the resultant thermo-chemical degradation.

\section{Simulation Predictions}

The geometry used by the numerical model replicates the CFC sample used in the laser ablation experiment. To optimise the numerical model, rather than modelling an entire strip of CFC, the model only considers the region directly surrounding the point of laser ablation. Furthermore, in addition to modelling a representative ply layout the numerical model also considers the piece of CFC to be unidirectional, with the fibre axis orientated along the $\mathrm{x}$ axis. This may be a reasonable assumption to make if the damage depth is limited to the first few plies as the through thickness conductivity varies only slightly for multi directional CFCs. However on the other hand, contact regions between plies with different fibre orientation may have low conductivity and it influences the heat transport and associated damage. Therefore, the effects of the change in fibre orientation in subsequent plies have been studied in the numerical experiments.

For an accurate comparison with the experimental results, it is important that the dimension along the fibre in the model, matches that of the experimental sample. This is because of the close proximity of the heat source to these sample edges means that it is unlikely that these edges will be at a constant ambient temperature, and so the distance to the boundary will affect the temperature profile within the material. The length of the geometry along the fibre direction $L_{x}$ is taken as the length of the fibre within the CFC sample. Due to the quasi-isotropic layup of the CFC panel, the fibre direction on the top panel is at 45 degrees to the $\mathrm{x}$ direction, and so $\mathrm{L}_{\mathrm{x}}$ is the length of the fibres along this 45 degree angle, which equates to $L_{x}$ being $10 \mathrm{~mm}$. The thickness of the assembly $\left(L_{y}\right)$ is taken to be the same as that of the real 
CFC sample i.e. $5 \mathrm{~mm}$. The dimension in $\mathrm{z}$ direction is less important due to the poor thermal conductivity of the top ply in these directions, which restricts the heat transfer. But subsequent plies have fibres along z direction and the sample length along this direction $\mathrm{L}_{\mathrm{z}}$ is taken to match that of $\mathrm{L}_{\mathrm{x}}$. The geometry used within the numerical model is shown in Figure 3.

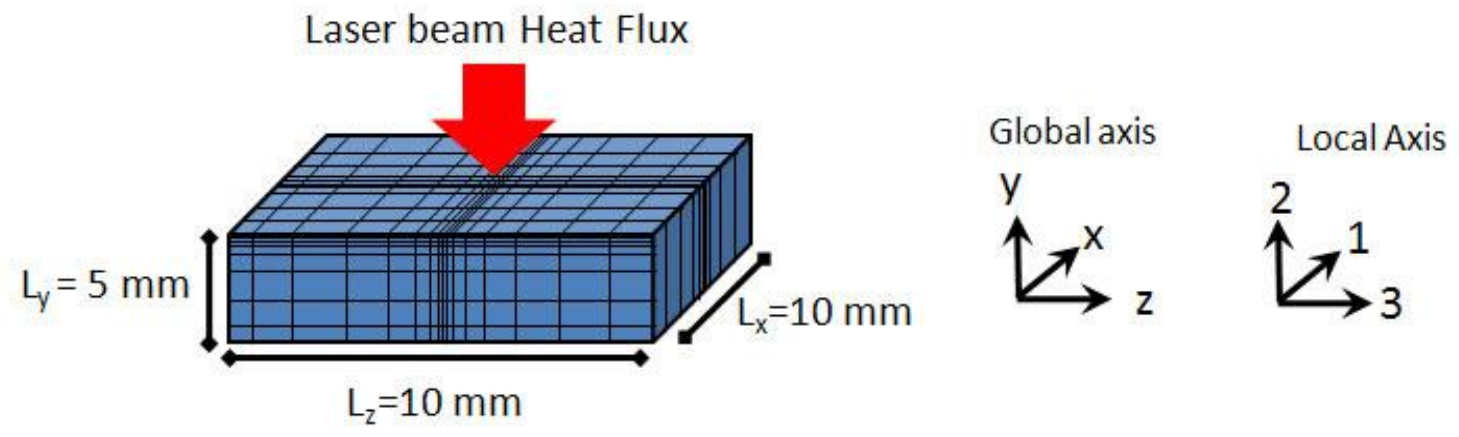

Figure 3: Geometry used by the numerical model

The laser heat flux defined by Equation (16) is positioned in the centre of the top surface as shown in Figure 3.

The gas transport Equation 11, 14 and 15 imposes strict conditions on time steps and these need to be considered. The time step may have a noticeable influence on temperature profiles, if not, then gas transport can be decoupled from pyrolysis. If the pressure build up is mainly linked to the void formation, it can be used to predict the fracture region. The numerical model is first used to investigate the details of the physical processes by considering a one dimensional degradation through the thickness the CFC. It represents a damage which takes place in CFCs under influence of powerful (several $\mathrm{kW}$ ) laser beam with diameter $(\sim 50 \mathrm{~mm})$ significantly larger than the panel thickness $5 \mathrm{~mm}$. Having investigated the physical processes, the simplified 3D model is then used to validate the numerical model by comparing the experimental results with numerical predictions.

\subsection{Qualitative prediction from 1D model}

The uniform heat flux of $0.38 \mathrm{MW} \mathrm{m}^{-2}$ is applied to the top surface of the panel. The system of equations described in section 3.1 is solved by the algorithm described in section 3.2. The non-uniform mesh was used where the size of the mesh element follows an arithmetic growth rate given by the $\mathrm{d}_{\mathrm{y}}$. For the purpose of this study the growth rate is defined by the size of the initial mesh element. A series of convergence tests for this model have been reported in [17] and have revealed that the temperature is calculated to less than $0.1 \%$ of a well defined analytical solution. 


\subsubsection{Pressure Effects of Gas Transport}

The temperature profile and the polymer volume fraction after $0.5 \mathrm{sec}$ and $1 \mathrm{sec}$ are shown in Figure 4. It can be seen that there is a definite change in slope of the temperature profile. This is a point where a sudden decrease in polymer volume fraction takes place over a particular time instance, which will be referred to as the reaction front. This change in temperature gradient is related to the variation of material properties due to the chemical degradation. . As the polymer is removed, thermal conductivity of the composite drops from $0.246 \mathrm{~W} /(\mathrm{mK})$ to $0.062 \mathrm{~W} /(\mathrm{mK})$. This initial analysis neglects variations in the polymer's properties due to pressure changes and glass transition (M21 $\mathrm{T}_{\mathrm{g}}$ is $383 \mathrm{~K}$ ) since the exact dependences are not known. Qualitatively, the conductivity of polymer may increase by factor of 2 [18] which would results in composite thermal conductivity of $0.484 \mathrm{~W} /(\mathrm{mK})$, at polymer temperatures above its $\mathrm{T}_{\mathrm{g}}$ of $383 \mathrm{~K}$. This would make the temperature profile in undamaged CFC flatter and the change in the slope would be even more pronounced.

\section{Uniform \\ Heat flux \\ Thermal insulation at edges}

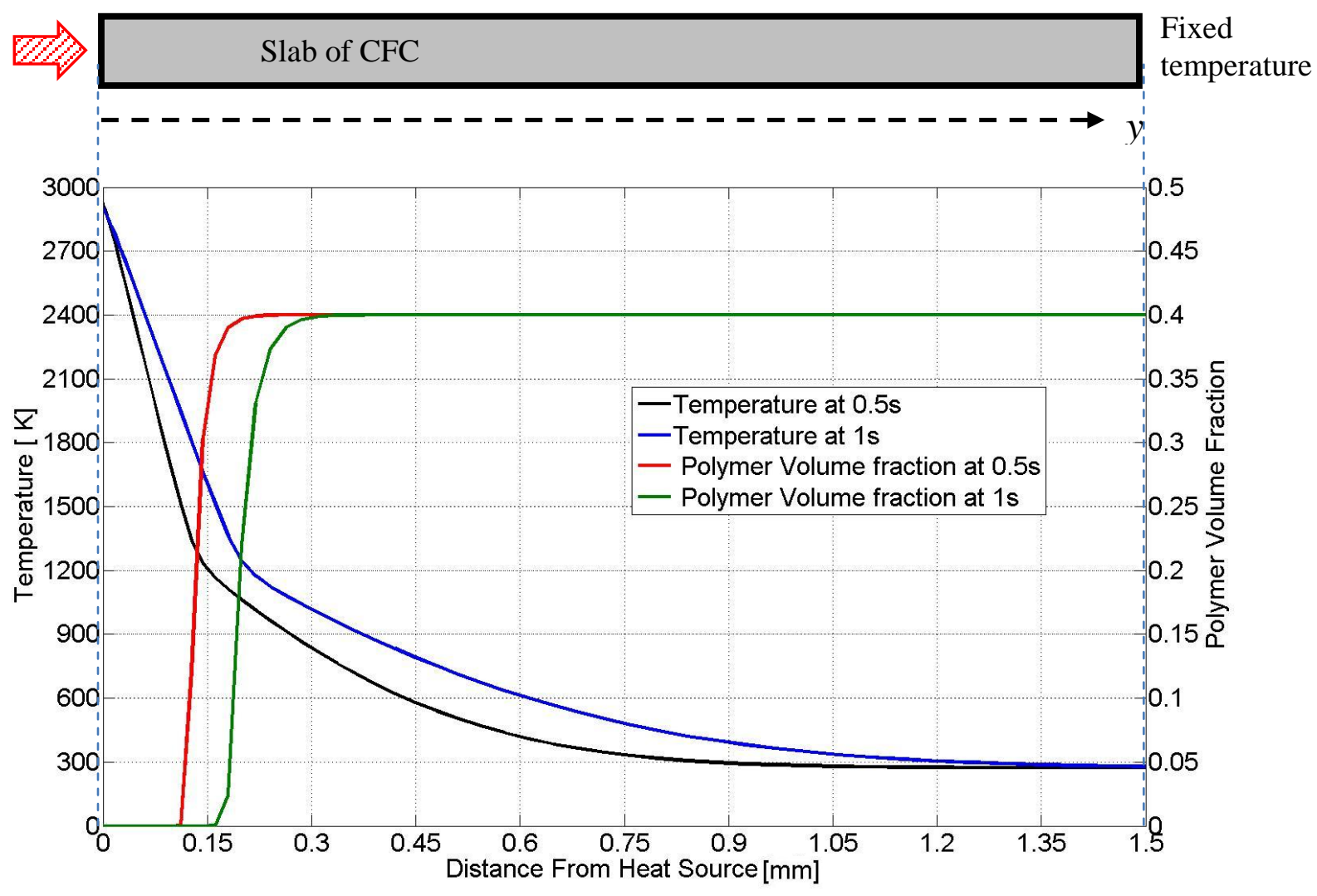

Figure 4: Through thickness temperature profile and polymer fraction predicted by the 1D model 
It is further evident from Figure 4 that the reaction front is relatively steep, which implies that the reaction occurs suddenly when the temperature of the polymer reaches a critical value, in this case approximately $1200 \mathrm{~K}$. It may appear that instead of modelling pyrolysis, it is possible to introduce a sublimation phase change at that temperature. Unfortunately, the kinetics of pyrolysis reaction are important, as the critical temperature at the reaction front depends on the input heat flux dynamics and it cannot be set a priori.

The gas accumulation within the material is represented by an effective gas density rather than the trapped gas mass within each mesh element, because due the non-uniform mesh it is more appropriate to compare the mass of gas per unit volume of the material. The effective gas density $\left(m_{p}^{\dagger}\right)$ is defined by

$$
m_{g}^{\dagger}=\frac{m_{g}}{V_{\text {cell }}}
$$

Equation 19

The effective gas density (Figure 5) shows an initial increase up to the reaction front, where it peaks. After this peak, there is a strong decrease in gas density followed by another peak.

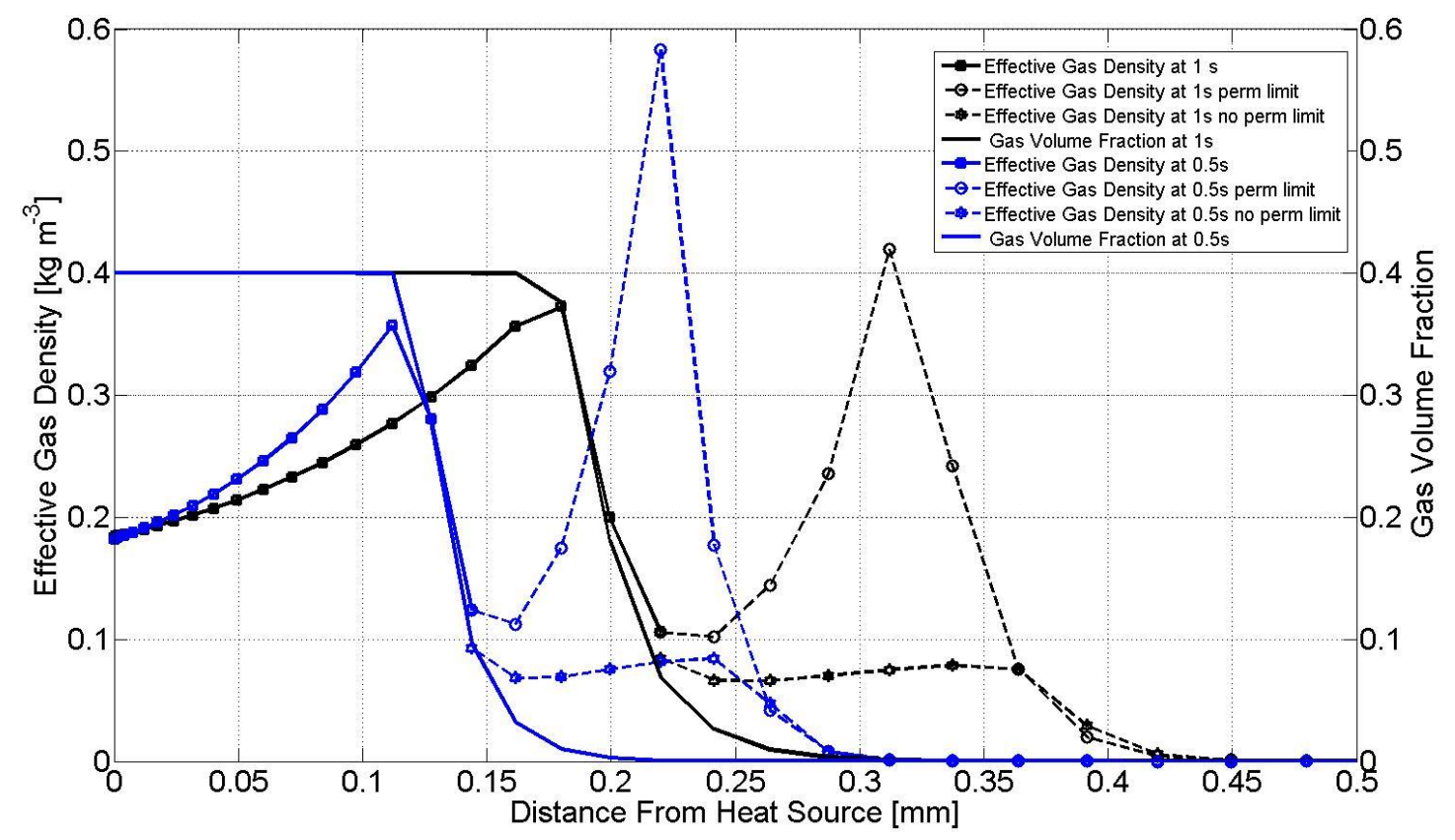

Figure 5: Effective gas density, solid lines - predictions up to a "realistic" limit of the model, dashed lines show the differences in predictions for $\sigma_{\max }=610^{7} \mathrm{~Pa}$, and for $\sigma_{\max }=\infty$ (no fracture)

The first increase in effective gas density is due to a large gas production in the vicinity of the reaction front. The gas filtrates through the material and escapes at the surface with the pressure drop 
between the front point and the surface. Moving deeper into the material, the gas production drops due to the decrease in temperature. However at some point, the amount of gas trapped inside begins to increase again if the permeability constraint Equation 16 is applied to avoid an over pressure. The second peak corresponds to very low porosity and almost zero permeability. Nevertheless, pyrolytic reaction still takes place here, which hence causes a very high pressure region, as shown in Figure 6.



Figure 6: Internal gas pressure and gas volume fraction, solid lines - predictions up to a "realistic" limit of the model, dashed lines show the differences in predictions for $\sigma_{\max }=610^{7} \mathrm{~Pa}$, and for $\sigma_{\max }=\infty$ (no fracture)

It is worth noting that the model describes this peak pressure region only qualitatively. The gas pressure in this region is above the mechanical strength of the polymer matrix and so would result in local fractures. As a result of the fracture the pressure drops not only because of increased filtration but also simply by availability of the additional volume for the gas to occupy. In summary, the second effective gas density peak in Figure 5 is due to permeability constraint Equation 16 applied to avoid an over pressure. We consider it as a model artefact, as in reality it should not be there due to the void formed by the fracture. It is solely due to the gas filtration from the bulk material with constrains imposed by Equation 16. If no fracture is assumed this second peak does not appear, see Figure 5. The model predictions in the vicinity of the second peak and to the right of it cannot be used for quantitative analysis. In fact the description of this region is limited by the conservation of mass and energy only and it is shown by dotted lines in Figures 5-7. 


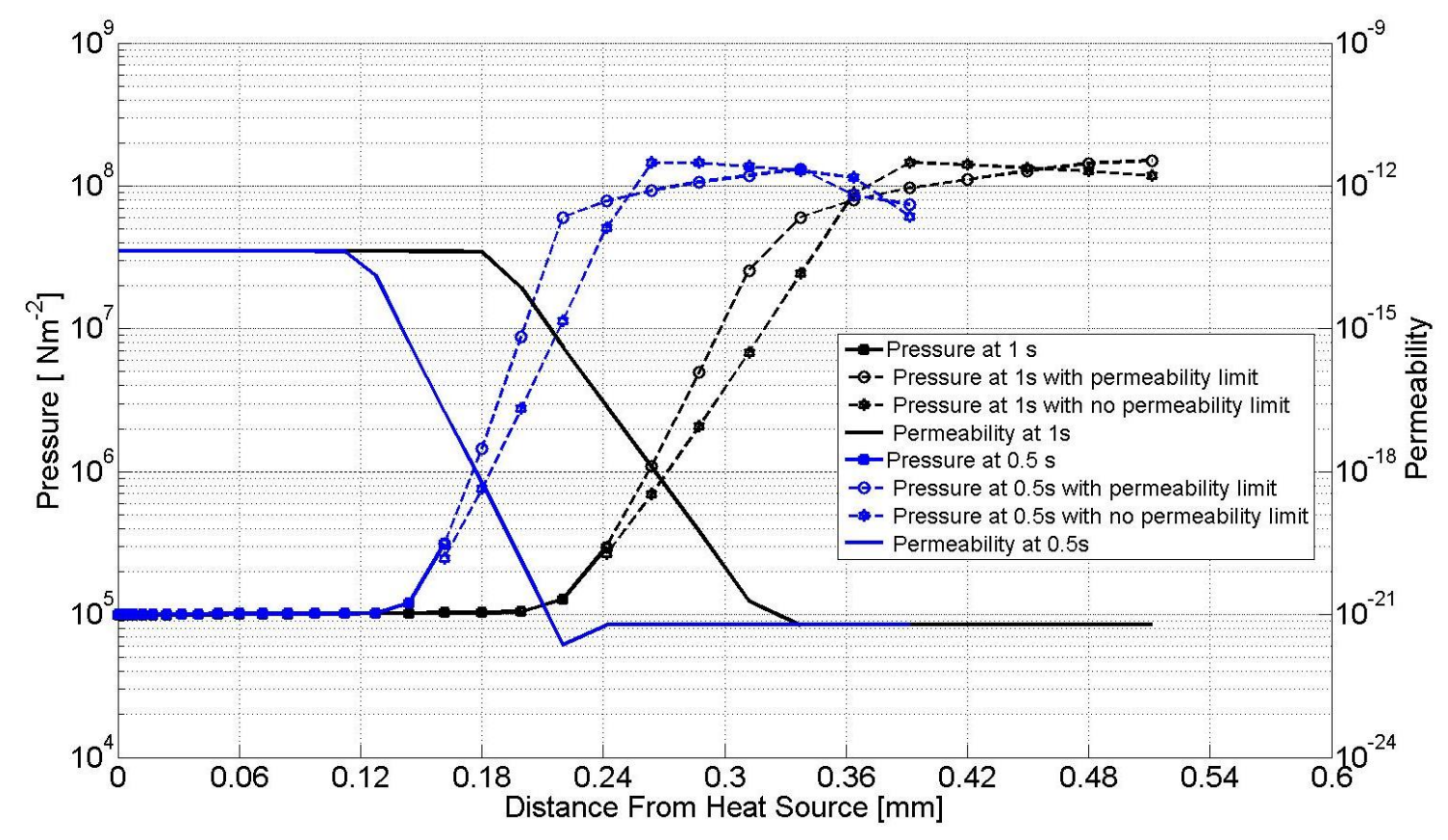

Figure 7: Pressure inside the decomposing material and its permeability as a function of the distance from the surface, solid lines - predictions up to a "realistic" limit of the model, dashed lines show the differences in predictions for $\sigma_{\max }=610^{7} \mathrm{~Pa}$, and for $\sigma_{\max }=\infty$ (no fracture)

The pressure profile portrays three distinct regions, which each can be explained with reference to Figures 5, 6, 7. Consider the pressure after $1 \mathrm{sec}$ of laser exposure and move from the heat source on the surface through the bulk of the CFC, the initial region shows an apparent constant pressure. There is in fact a slight increase in pressure from the boundary to approximately $0.2 \mathrm{~mm}$. The slight increase in pressure is caused by the increasing effective gas density which overcomes the decrease in temperature. After this initial region, there is a large increase in gas pressure between $0.2 \mathrm{~mm}$ and $0.33 \mathrm{~mm}$ in depth. This several orders of magnitude increase is caused by the gas volume fraction tending towards zero and low permeability not allowing this gas to escape. Gas staying at almost solid density would expect to have a very large pressure (of course the ideal gas law is a very crude approximation for such conditions and can be used only for qualitative analysis). By comparing Figures 5 and 6 , it is evident that as the gas volume fraction decreases there is a rapid decrease in permeability until the permeability becomes constant due to local fractures at around $0.33 \mathrm{~mm}$ depth. The low volume fraction of gas $(5 \%)$ can be defined with reference to the reaction front at $0.23 \mathrm{~mm}$. It is observed that the fracture pressure occurs at around $0.35 \mathrm{~mm}$, far ahead of this reaction front with the volume fraction of gas been very small $(0.05 \%)$. The peak internal gas pressure in Figure 6 is approximately $2 \times 10^{8} \mathrm{Nm}^{-2}$, which is above the tensile strength of the polymer, occurs when the void volume fraction is below $0.01 \%$. It is a non-realistic 
prediction and it is shown in the figure as a dashed line. It allows the gas pressure to exceed the tensile strength in this calculation assuming constant permeability in this region. The fracture opens the pores, releases the gas but also reduces the pressure by increasing void volumes, although such pressure changes were not incorporated into the model. It was not included because in reality for small voids the local compressibility would compress the surrounding polymer and the real pressure would drop to $\sigma_{\max }$ $\left(6 \times 10^{7} \mathrm{~Pa}\right.$ in our model) without any fracture and associated increase in the permeability. Approximately, based on the combination of model predictions and the experiment (Figure 2) it can be assumed that the fracture occurs at the point where the volume fraction of voids exceeds $0.05 \%$.

\subsubsection{Thermal Convection Effects of Gas Transport}

The influence of the convective gas transport on the internal energy of the system has been investigated. For comparative analysis the same 1D model as used in section above is re-solved, with and

without the transport model subroutine, term $-\nabla\left(v\left(\rho_{g} C_{v g} T+p\right)\right)$ in Equation 11. Any excessive gas (above atmospheric pressure) produced in the pyrolysis process had been removed from the cell and for material properties calculations it was assumed that the gas in voids stays at 1atm. The numerical predictions from these models are compared by considering the difference in the mass of degraded polymer $(\Pi)$, which is defined by

$$
\Pi=\left|\left(\Sigma m_{p}\left(t_{0}\right)-\Sigma m_{p}(t)\right)-\left(\Sigma m_{p}\left(t_{0}\right)-\Sigma m_{p}^{*}(t)\right)\right| \quad \text { Equation } 20
$$

where, $\Sigma m_{p}\left(t_{0}\right)$ and $\Sigma m_{p}(t)$ are the summation of the total polymer mass at the initialisation of the model and later at time $t$. The superscript " ' refers to the model which included the gas transport. The difference of the degraded polymer, shown in Figure 8, highlights any differences in temperature profile which would have affected the chemical reaction. 


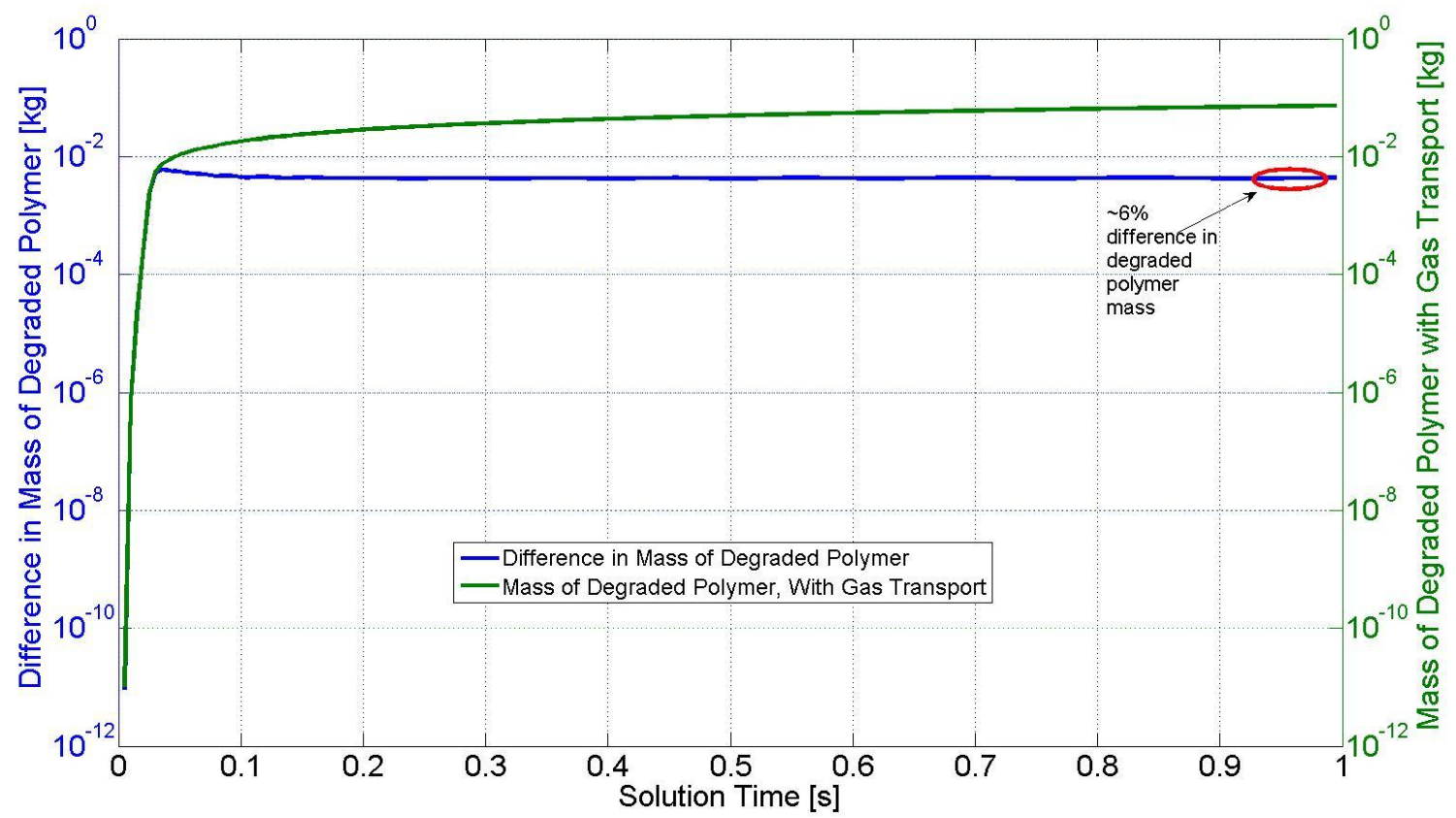

Figure 8: Difference in the amount of polymer mass which degraded when the gas transport has been included and omitted. The difference increases up to $0.03 \mathrm{~s}$, however after this point there are no noticeable changes. The maximum difference in degraded polymer mass is about $5 \cdot 10^{-3} \mathrm{~kg}$ which equates to approximately $6 \%$ variation between models.

From Figure 8, it is evident that for solution times above $0.03 \mathrm{sec}$, there is a roughly constant $6 \%$ difference in the amount of polymer which has degraded. This slight difference can further be seen in temperature profiles predicted by these two models, as shown in Figure 9. The gas convection slows down the propagation of the decomposition front at the initial stage up to $0.1 \mathrm{sec}$ and this slight delay stays intact at later stages. The gas convection slows down the propagation of the decomposition front at the initial stage up to $0.03 \mathrm{~s}$ and this slight delay stays intact at later stages. Generally speaking the gas transport needs to be included when the surface ablation plays a major role in the damage. In the case of lightning strike the degradation takes place deep inside the material (hundreds of microns), and in this case neglecting of gas transport effects is a reasonable simplification. 


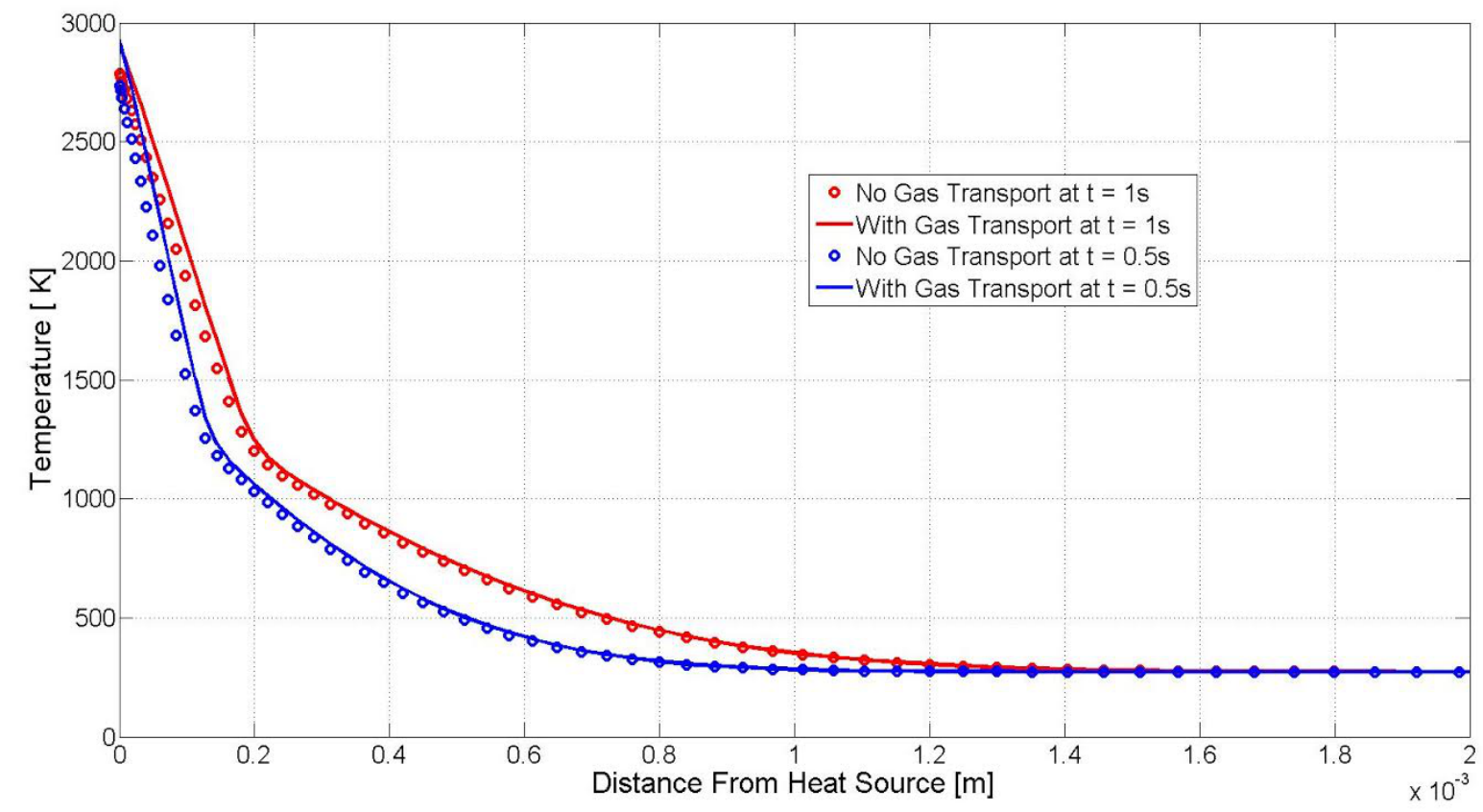

Figure 9: Temperature profiles at two different time instances for the model with and without the gas

transport

\subsubsection{Discussion}

Numerical predictions from the 1D model have shown several distinguishing features of the degradation process. Principal among these is that Figure 6 demonstrates, that the peak internal gas pressure is large enough to cause de-lamination and cracking. Furthermore it appears that the magnitude of the peak pressure is defined by the permeability of the system, as it restricts the movement of gas from the large pressure regions. The quantitative description of the fracture requires modelling of polymer compressibility, local fracture and complex variations in permeability. The current model formulation does not have these features and it can be used only for qualitative predictions of the extent to which the fracture occur. Nevertheless, the model suggests that the high pressure region extends approximately two times further than the reaction front and can be approximately described as a gas volume fraction of 0.0005. It has also been shown that thermal gas transport has minimal affect on the predictions of temperature profile. The removal of the thermal gas transport model dramatically reduces the numerical solution time, so realistic predictions can be obtained by omitting gas convection from the model. Instead, the fractured region is approximately described by a contour where the gas volume fraction is $\varphi_{\mathrm{g}}=0.0005$.

\subsection{Full 3D modelling of Thermal Degradation of CFC Panels}

To draw a comparison between the experimental laser ablation data and the $3 \mathrm{D}$ model the 
simulation was conducted without gas convection as justified in the previous sub-section.

\subsubsection{Extend of Polymer Degradation}

To compare the experimental results with numerical predictions, three parameters were analysed. The damage was measured in terms of polymer removal. The extent of polymer degradation on the top surface along the fibre direction, normal to the fibre direction (which are termed the major and minor axis, respectively) and the maximum polymer degradation depth are the 3 parameters used to describe the damage. These damage dimensions are assigned to the numerical predictions of the lengths at which the polymer volume fraction has fallen to below $5 \%$ of its initial volume fraction i.e. $\phi_{p}=0.05 \phi_{p}\left(t_{0}\right)$. Although such choice of the critical polymer volume fraction is a bit arbitrary, it is not critical given the sharp reaction front as seen in Figure 5. The comparison is first drawn by considering the predicted damage along the major and minor axes for the polymer degradation, shown in Figure 10 and Figure 11. The raw numerical predictions show a step like profile, which is solely the effect of homogenization in a single cell. The mesh size used is $50 \mu \mathrm{m}$ by $50 \mu \mathrm{m}$ by $25 \mu \mathrm{m}$, which is approximately equal to the width of the decomposition front. At a particular time instance the first half of the cell is fully decomposed while the second half still contains only slightly damaged composite. It means that the realistic predictions for the damage extent can be obtained by joining together the leading edges of each steps as shown in Figures 10 and 11. Numerical simulations with the reduced mesh sizes confirmed this prediction. 


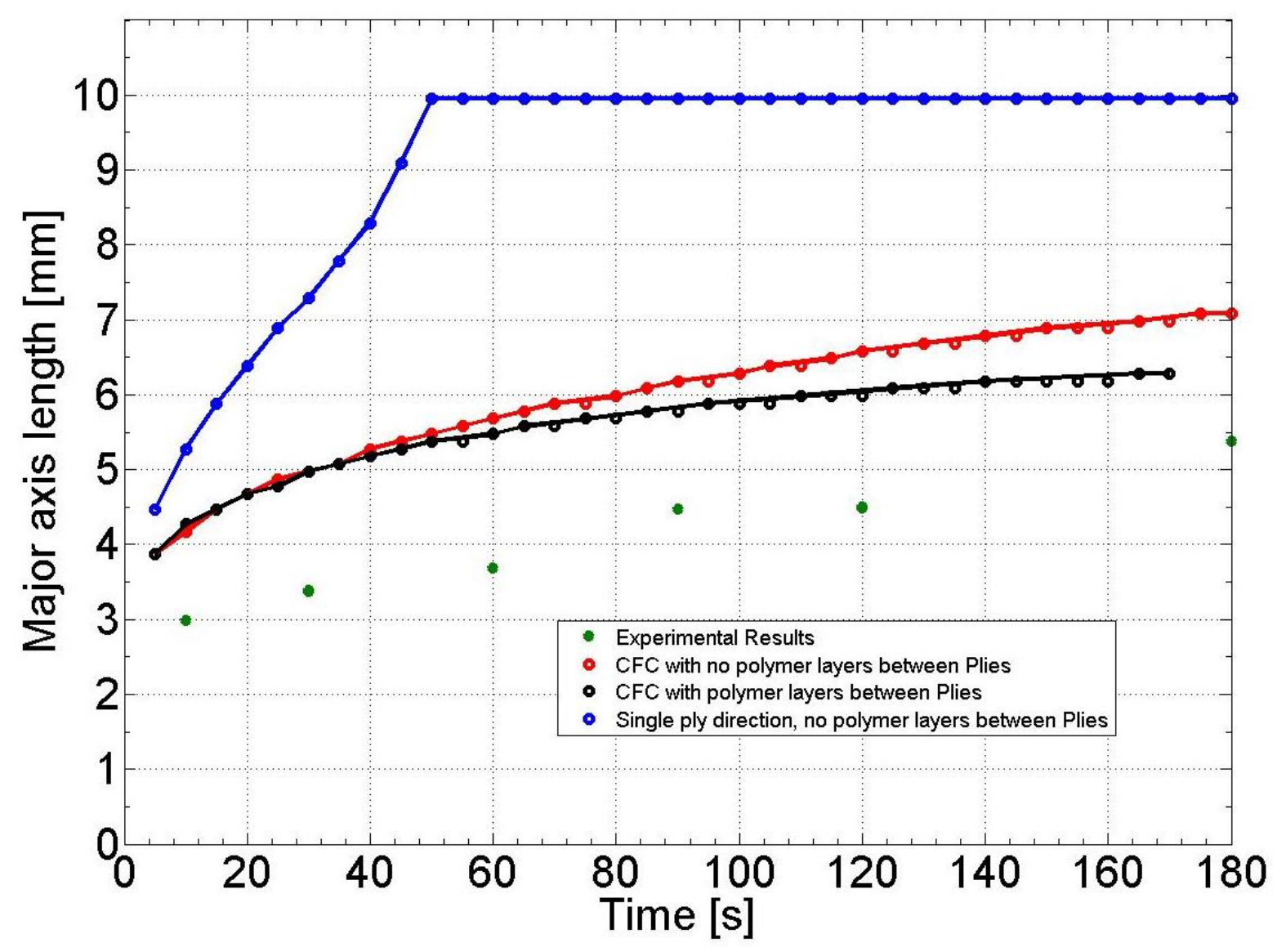

Figure 10: Comparison between predictions of the thermo-chemical degradation and experimental laser ablation results for the damage along the major axis. Adding or removing polymer layers between the plies has a small effect on the damage, whereas the plies orientation has a strong effect. 


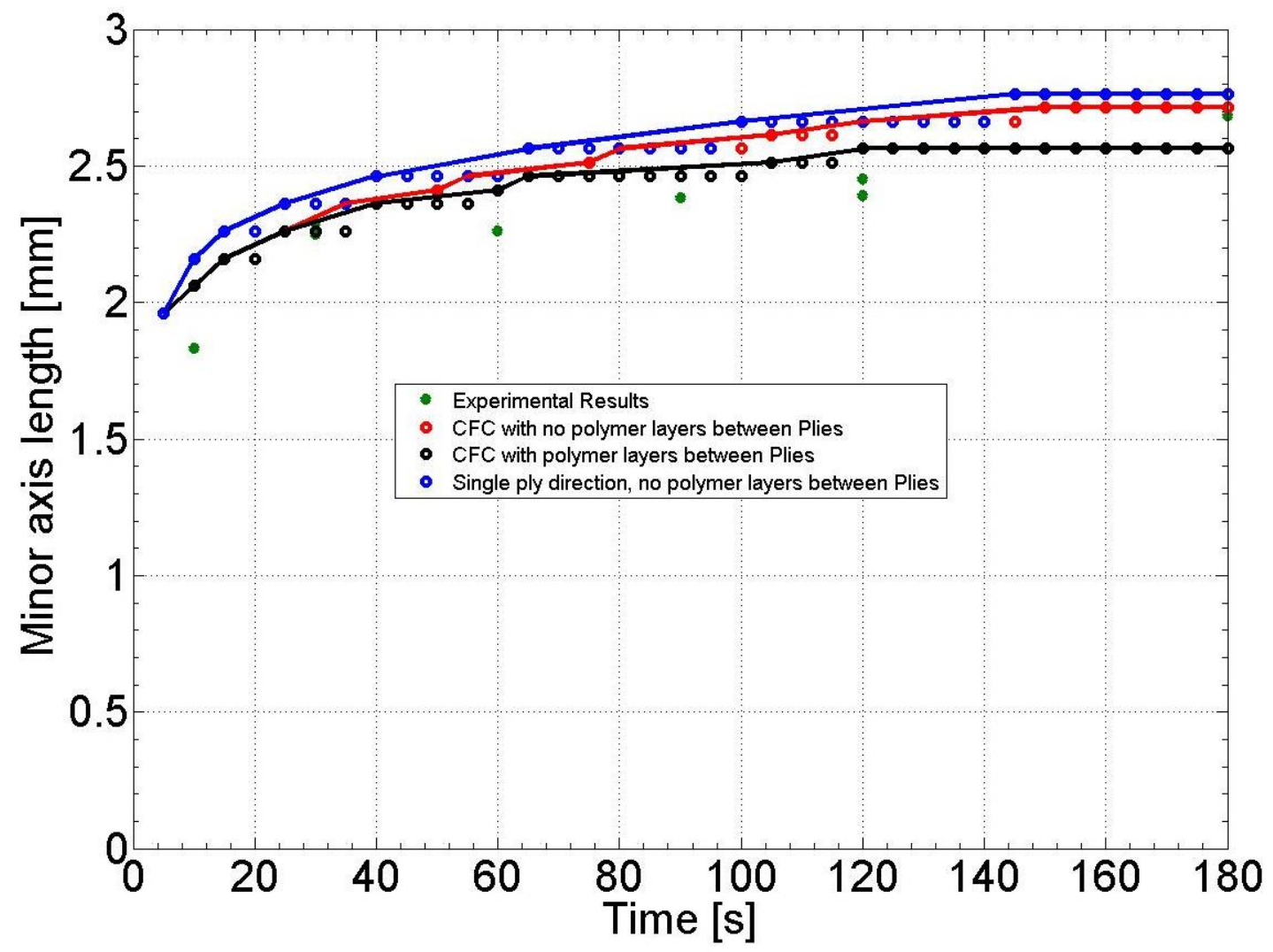

Figure 11: Comparison between predictions of the thermo-chemical degradation and experimental laser ablation results for damage along the minor axis. The damage is approximately within the laser beam diameter.

The numerical predictions for the major and minor axes both show an increasing trend over time, with the major axis showing a greater rate of increase, which is expected. The higher thermal conductivity along the fibres directs the heat flow along the major axis and this excessive heat drives polymer pyrolysis. The predicted damage along the major axis for the unidirectional CFC sample reaches saturation at $10 \mathrm{~mm}$, which is the size of the model panel. A similar comparison was undertaken for the maximum damage depth, as shown in Figure 12. It is obvious that changes in fibre orientation between the plies significantly reduces the damage, not only along the fibres but also through the thickness of the CFC plaque. 


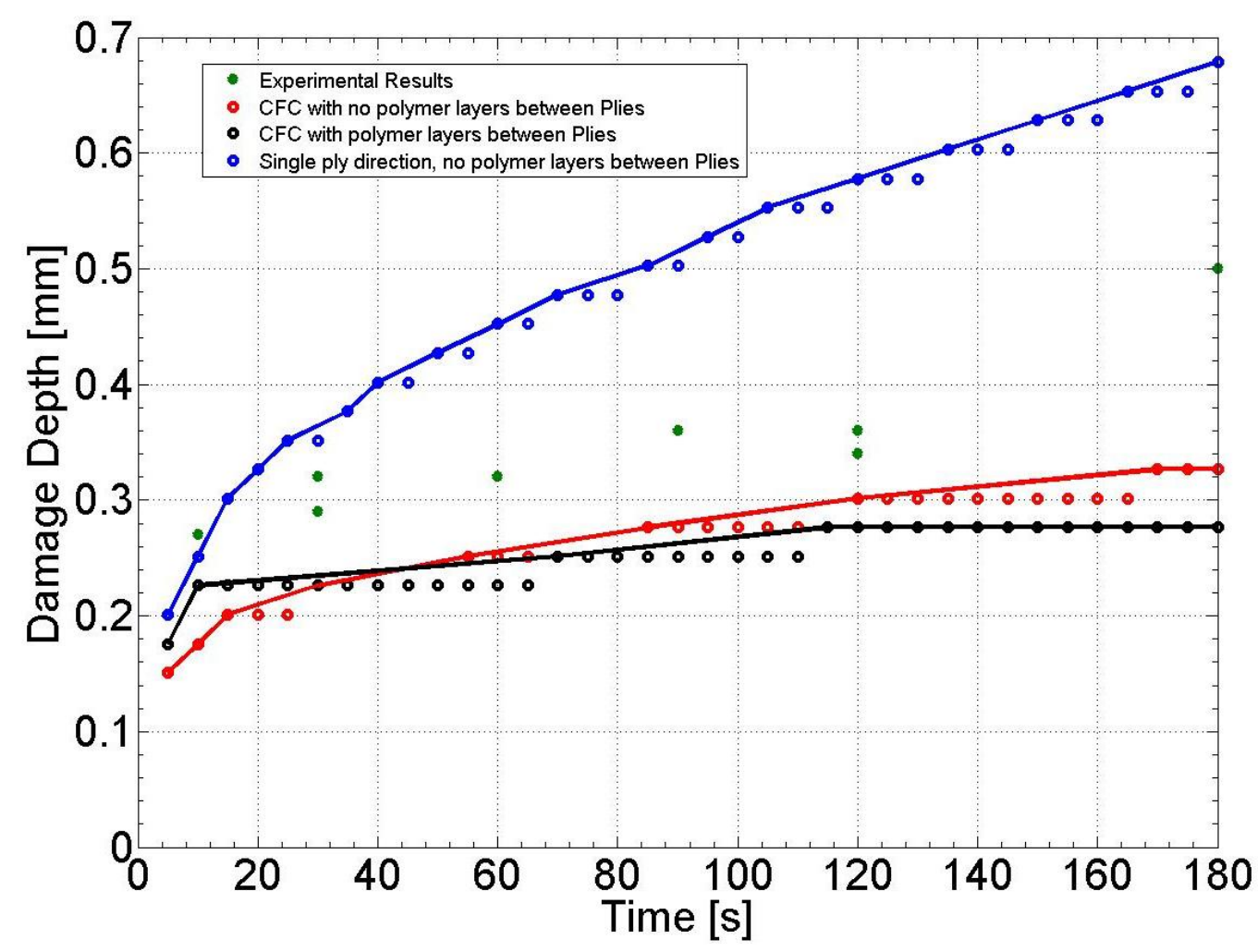

Figure 12: Comparison between predictions for the thermo-chemical degradation and experimental laser ablation results for the damaged depth.

By comparing the numerical predictions with the experimental results it is evident that the damage length along the minor axis is in a very good agreement with the experimental results. Since the heat mainly dissipates along the fibres, the damage is approximately within the laser beam diameter. On the other hand, the numerical predictions overestimate the measured damage along the major axis but underestimate the experimentally observed damage depth. This is mainly due to uncertainties in the material properties, especially their dependence on temperature. Sensitivity studies [17] showed that the realistic variation in thermal properties may change the damage extent by the factor of 2 . The leading discrepancy between the predictions and the measurements come from the initial stage of the ablation process, the rate at which both the predicted major length and the damage depth increase is roughly comparable to that of the experimental rate. This could imply that the specific heat values used in the model are not accurate. Another reason which might explain the differences is that the numerical model did not consider carbon fibre removal. If carbon fibres were removed then the heat flux boundary would be at a deeper depth within the material, which would certainly produce a greater damage depth. It would also reduce the thermal input to the fibre in the top layer and decrease the damage along the major axis. 
Modern CFCs panel contain thin polymer layers between the plies. Modelling of such layers would need a relatively fine mesh. The simulation suggests that in many cases these layers can be omitted from the model. The model predictions are affected only slightly by these layers, as shown in Figures 10 , 11 and 12.

Comparison between multi-directional CFC panel and unidirectional cases shows a significant difference. Although it is simpler to consider a unidirectional CFC panel (or indeed prepare experiments with such panels), such damage predictions are an overestimate of the experimentally measured damage and so it is recommended to use the real ply layup. This result further indicates that the replacement of 3D composite structures by effective 2D axisymmetric model [6] cannot predict the experimental features shown in Figure 1.

\subsubsection{Model Sensitivity to Variations in Materials Properties}

The model assumes temperature and pressure independent values for thermal conductivity, specific heat and density. Possible effects of these simplifications have been briefly mentioned in sections 2.3 and 5.1.1. The sensitivity of the numerical predictions to the material properties has been analysed. The thermal conductivity of the polymer is the most variable parameter which may probably vary $-25 \%$ to $+100 \%$ [18]. Expected changes in volumetric specific heat are smaller, 25\% increase at high temperature and pressure is a reasonable assumption [18]. The model predictions are shown in Figure 13 and Figure 14.

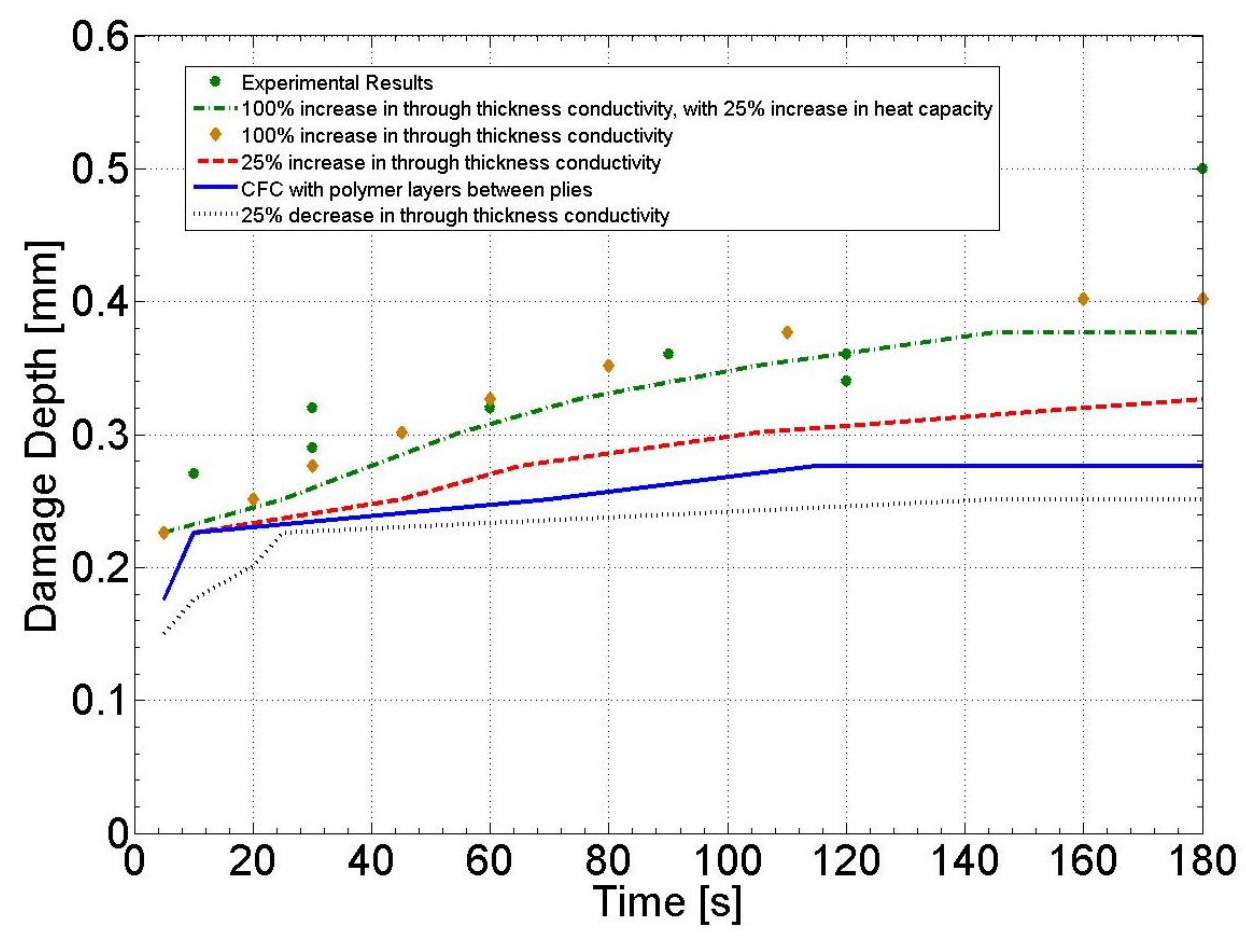

Figure 13: Comparisons of the polymer degradation depth as the through thickness conductivity is 
varied in $-25 \%-+100 \%$ range in respect to reference value calculated using Eq.(7) with input given in Table 1 . The model geometry includes polymer layers between plies in CFCs.

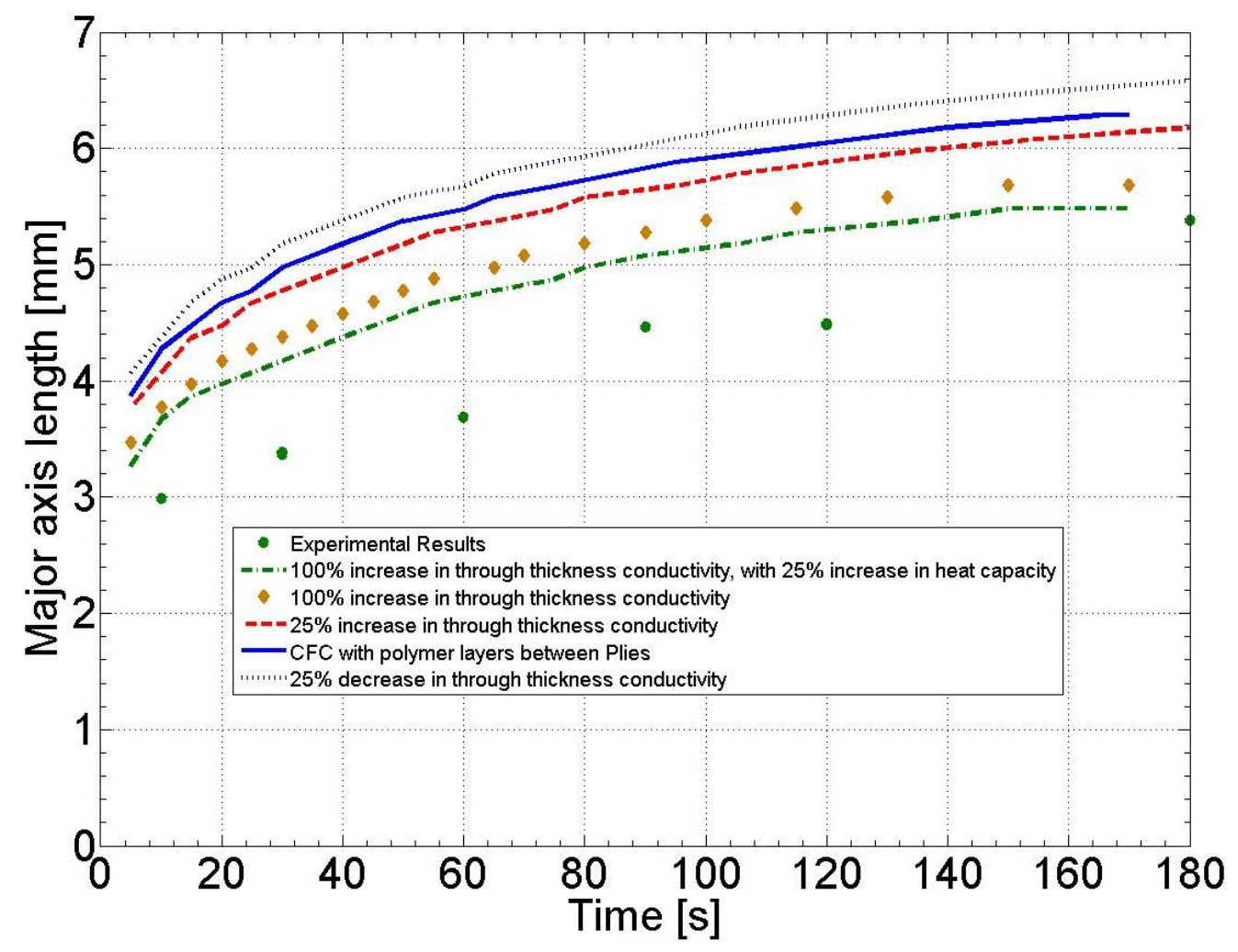

Figure 14: Comparisons of polymer degradation along the major axis as the through thickness conductivity is varied in $-25 \%-+100 \%$ range in respect to reference value calculated using Eq.(7) with input given in Table 1. The model geometry includes polymer layers between plies in CFCs.

From the above figures it is evident that as the through thickness conductivity is increased, there is a noticeable increase in damage depth and a slight decrease in damage along major axis. This can be explained by the increased heat flux towards the material depth as through thickness conductivity increases, at the cost of heat flux propagating along the fibre direction. There is approximately a linear variation in damage dimensions with the thermal conductivity within the range considered and the increase in the conductivity of polymer makes an agreement between the model and the experiment better.

The increase in volumetric specific heat of the polymer changes the predictions very little. Actually variation of these material properties only affects the initial transient increase of polymer degradation. 


\subsubsection{Fracture and Delamination}

Components of modern CFCs are specially selected to reduce any thermal strains which may appear due to temperature variations i.e. differential thermal expansion. It means that even high thermal gradients in the ablation experiments would not introduce large mismatch strain between the plies and it should not lead to the observed delamination. On the other hand, the gas generation and associated high pressure may lead to the local fracture, as shown in Figure 2. Section 5.1.3 indicates that the pressure required for the fracture can be built up at the point where the gas volume fraction content is as low as $\varphi_{\mathrm{g}}=0.0005$. Comparison between Figure 15 and Figure 2 indicates that it is reasonable to assume that the extent of fractured region can be predicted by the gas volume fraction. Furthermore Figure 13 also indicates that due to the presence of polymers layers between plies, the pressure build is elongated in these regions and under-cuts the CFC layers.

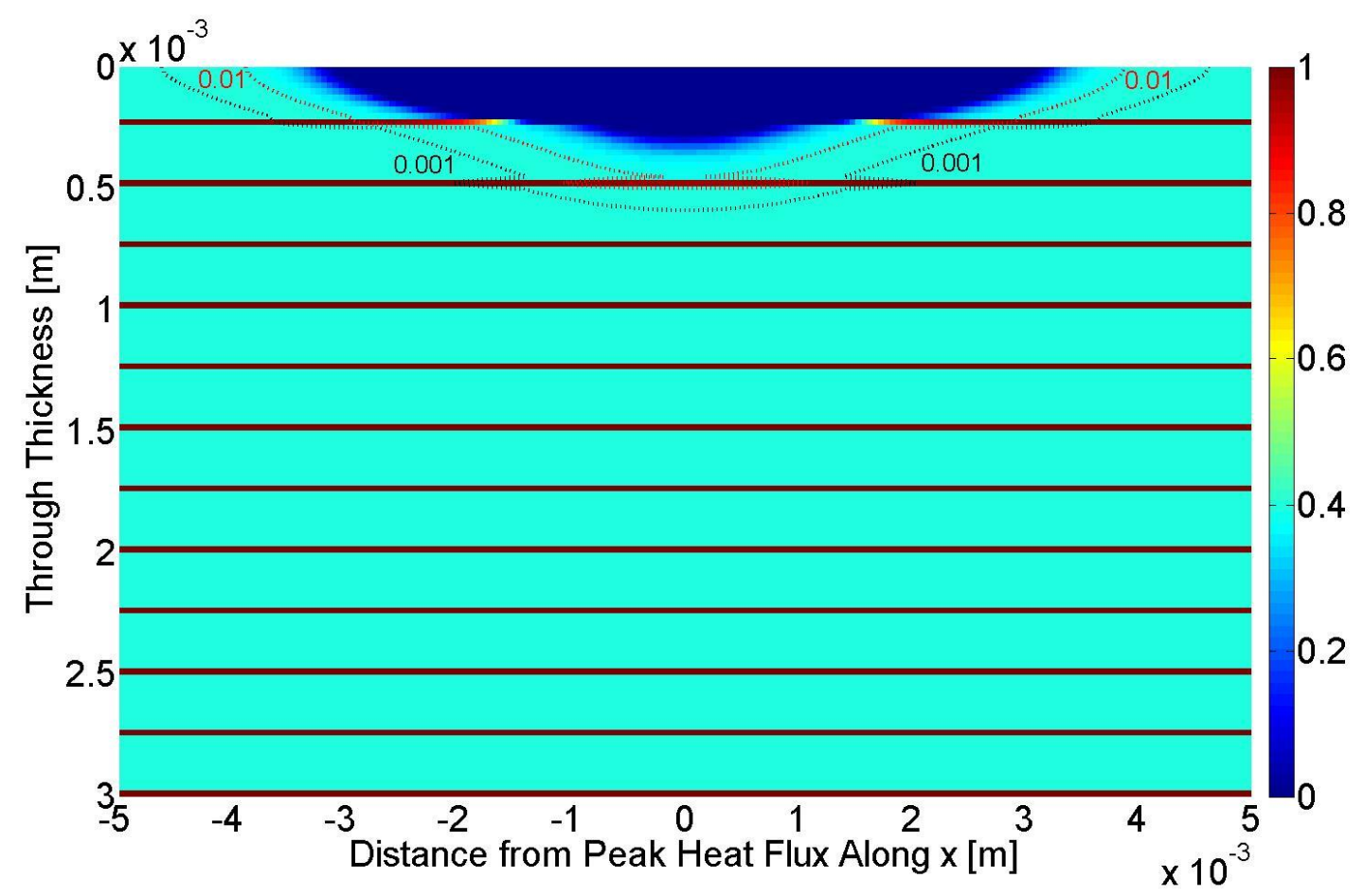

Figure 15: The cross-section of the model sample with polymer volume fraction shown. Also the isolines of the constant polymer volume fraction are shown. The high pressure region approximately extends to $\varphi_{\mathrm{g}}=0.0005$. Note that the fracture may occur far ahead of the degradation front.

\section{Conclusions}

A thermo-chemical degradation model has been used to analyse a complex damage in CFCs under intensive heat fluxes typical for lightning strikes, fires or laser processing. The laser ablation experiment was chosen as a verification tool since it simplifies the inputs required by the model, and it restricts the 
consideration to a thermal model only with no need for an electrical conduction model. The model assumptions and input parameters were verified by the experiments. It was shown the model successfully describes both the polymer pyrolysis and the high gas pressure produced in such decomposition. The observations can be summarised as follows:

- The observed damage mainly consist of polymer decomposition and delamination with small amount of carbon fibres being removed. The polymer degradation shows a clearly noticeable front between virgin $\mathrm{CFC}$ and bare fibres.

- Fractures have been found at significant distance in front of the reaction front. Since components of modern CFCs are specially selected to reduce any thermal stresses, the observed fractures are believed to be a result of the gas products from the pyrolysis.

- The developed model also suggests that the main reason for the delamination is a high pressure of the gas products of the polymer decomposition. The extent of the fracture is linked with tensile strength of the polymer.

- For a particular composite the fracture conditions can be approximately attributed to the minimum voids volume fraction which leads to such fracture, e.g. for M21/T700 composites this value is about 0.0005. Since the critical volume fraction is very small, the delamination can be seen at significant distances in front of the reaction front.

- The distribution of voids and temperature profile within the composite panel can be predicted from convection free formulation.

- The anisotropy and layup of the plies within the composite panel are very important for heat transport. Common simplifications like axisymmetric in-plane approximation or a single unidirectional layer lead to erroneous predictions of the damage topology and extent.

\section{Acknowledgements}

This work has been supported by EADS Innovation Work, through an industrial studentship. The authors gratefully acknowledge the $\mu$ VIS centre at the University of Southampton for provision of tomographic imaging facilities, supported by EPSRC Grant EP-H01506X. The authors are also grateful to Dr. Matthew Cole for various useful discussions and to Prof. James Wilkinson for his guidance on experimental laser ablation facilities.

\section{References}

[1] B. Reid and G. W. Clarke, "Further Investigations into the Damage of Various Types of Unprotected Carbon Fibre Composites with a Variety of Lightning Arc Attachments," Proceedings of the 1994 
international aerospace and ground conference on lightning and static electricity, pp. 193-205, 2427 May 1994, Mannhiem, Germany.

[2] A. P. Mouritz, S. Feih, E. Kandare, Z. Mathys, A. G. Gibson, P. E. D. Jardin, S. W. Case and B. Y. Lattimer, "Review of fire structural modelling of polymer composites," Composites Part A: Applied Science and Manufacturing, vol. 40, no. 12, pp. 1800 - 1814, 2009.

[3] A. Wolynski, T. Herrmann, P. Mucha, H. Haloui and J. Huillier, "Laser Ablation of CFRP Using a Picosecond Laser Pulses at Different Wavelengths From UV to IR,” Physics Procedia, vol. 12, pp. 292-301, 2011.

[4] G. Jiang, S. Pickering, G. Walker, N. Bowering, K. Wong and C. Rudd, "Soft ionisation analysis of evolved gas for oxidative decomposition of an epoxy resin/carbon fibre composite.," Thermochimica Acta, vol. 454, no. 2, pp. 109-115, 2007.

[5] J. Mathew, G. Goswami, N. Ramakrishnan and N. Naik, "Parametric studies on pulsed Nd:YAG laser cutting of carbon fibre reinforced plastic composites," Journal of Material Processing Technology, vol. 89, pp. 198-203., 1999.

[6] F. Lago, J. J. Gonzalez, P. Freton, F. Uhlig, N. Lucius and G. P. Piau, "A numerical modelling of an electric arc and its interaction with the anode: part III. Application to the interaction of a lightning strike and an aircraft in flight," Journal Of Physics D-Applied Physics, vol. 39, p. 2294 2310, 2006.

[7] N. Jennings and C. J. Hardwick, "A computational approach to predicting the extent of the arc root damage in CFC panels," 15th International Aerospace and Ground Conference on Lightning and Static Electricity, pp. 41.1 - 41.8, 1992, Atlantic City .

[8] C. Cheng, Y. Tsui and T. Clyne, "Application of a three dimensional heat flow model to treat laser drilling of carbon fibre composites," acta Mater, vol. 46, no. 12, pp. 4273-4285, 1998.

[9] D. Herzog and P. Jaeschke and O. Meier and H. Haferkamp, "Investigation on the thermal effect caused by laser cutting with respect to static strength of CFRP," International Journal of Machine Tools and Manufacture, vol. 48, pp. 1464-1473, 2008.

[10] W. S. O. Rodden, S. S. Kudesia, D. P. Hand and J. D. C. Jones, "A comprehensive study of the long pulse Nd:YAG laser drilling of multi-layer carbon fibre composites," Optics Communications, vol. 210, no. 4, pp. 319-328, 2002.

[11] M. DellErbaa, L. Galantuccib and S. Migliettaa, "An experimental study on laser drilling and cutting of composite materials for the aerospace industry using excimer and $\mathrm{CO} 2$ sources," Composites Manufacturing, vol. 3, pp. 14 - 19, 1992.

[12] A. Cenna and P. Mathew, "Evaluation of cut quality of fibre reinforced plastics-a review," 
International Journal of Machine Tools and Manufacture, vol. 37, no. 6, pp. 723-736, 1997.

[13] R. D. Chippendale, I. O. Golosnoy, P. Lewin and J. K. Sykulski, “Transport properties and current flow patterns in homogeneous strongly anisotropic materials," COMPEL: The International Journal for Computation and Mathematics in Electrical and Electronic Engineering, vol. 30, no. 3, pp. 10471055, 2011.

[14] J. W. Klett, V. J. Ervin and D. D. Edie., "Finite-element modeling of heat transfer in carbon/carbon composites.," Composites Science and Technology, vol. 607, p. 593-607, 1999.

[15] M. W. Pilling, M. A. Black and B. Yates, "The thermal conductivity of carbon fibre reinforced composites," Journal of Materials Science, vol. 14, p. 1326:1338, 1979.

[16] T. W. Clyne and D. Hull, An Introduction to Composite Materials, Cambridge University Press, 2nd edition, 1996.

[17] R. Chippendale, Modelling of the Thermal Chemical Damage Caused to Carbon Fibre Composites, University of Southampton, PhD thesis, 2013.

[18] B. Sundqvist, O. Sandberg and G. Backstrom, "The thermal properties of an epoxy resin at high pressure and temperature," J. Phys. D: Appl Phys., vol. 10, pp. 1397-1403, 1977.

[19] E. Farkas, Z. G. Meszena, A. Toldy, S. Matkó, B. B. Marosfoi and G. Marosi, "Modelling of transport processes in a developing char,", Polymer Degradation and Stability, vol. 93, no. 6, pp. $1205-1213,2008$.

[20] Toray CA," “ technical data sheet for T700s,” Toray CA,.

[21] J.D.Anderson, Fundamentals of Aerodynamics, McGraw-Hill, 3rd Edition, 2001.

[22] M. J. MacDonald, C. Chu, P. P. Guilloit and K. M. Ng, "A generalized Blake-Kozeny equation for multi sized spherical particles," AIChE Journal, vol. 37, no. 10, pp. 1583-1588, 1991.

[23] R. Diasa, C. Fernandesb, J. Teixeirac, M. Motac and A. Yelshinc, "Permeability analysis in bisized porous media: Wall effect between particles of different size," Journal of Hydrology, vol. 349, no. 3, pp. 470-474, 2008.

[24] D. A. Nield and A. Bejan, Convection in Porous Media, Springer, 4th Edition, 2002.

[25] N. N. Yanenko, The method of fractional steps: The solution of problems of mathematical physics in several variables, Springer, 1st Edition, 1971.

[26] Coherent Inc, "VERDI V2,V5 and V6 - Diode-Pumped Laser Technology,” 2003.

[27] A. Scott, M. Mavrogordato, P. Wright, I. Sinclair and S. Spearing, "In situ fibre fracture measurement in carbon-epoxy laminates using high resolution computed tomography," Composites 
Science and Technology, vol. 7, no. 12, p. 1471-1477, 2011. 